\title{
A FORMAL METHOD FOR ASSESSING PRODUCT PERFORMANCE AT THE CONCEPTUAL STAGE OF THE DESIGN PROCESS
}

\author{
PAUL RODGERS, ALISTAIR PATTERSON AND DEREK WILSON \\ University of Westminster, United Kingdom
}

\begin{abstract}
The paper describes a formal methodology for defining and assessing product performance and its implementation in a prototype computer system. The methodology is based on high level abstract descriptions of the operations conducted within the design process. It is consequently extremely generic and succeeds in formally bridging the gap between physical product performance and actual end-user requirements. The methodology is based on defining product attributes as observable behaviour of the product in use. Defining an attribute in this way inherently reflects its required interaction with the end-user and consequently can truly be said to be in "end-user terms". A product will have a range of attributes and a performance indicator is found by combining them in a way that reflects their relative importance to the end-user. At the conceptual stage of the design process, however, the actual product does not exist, only some representation of it. To assess products at this stage requires a model or simulation of its attributes. This methodology has been implemented in a prototype Computer Aided Design Evaluation Tool (CADET) and tested with an existing product range. An example of which is presented within the paper.
\end{abstract}

\section{Introduction}

Human beings have always designed things. One of the most elementary traits of human beings is that they make a wide range of artifacts and tools to suit their own needs. As those needs alter, and as artifact users' reflect on the currently-available artifacts, so refinements are made to the artifacts, and completely new kinds of artifacts and tools are created and manufactured (Cross, 1994).

In the past twenty years or so there has been a significant cultural change towards manufactured goods in that product designers and manufacturers have passed through the period in which it was a challenge to manufacture an artifact to one in which the challenge is to 'Design and Manufacture' a product that satisfies user needs, wants or desires (JIDPO, 1990).

Today the quality of many products reaches such a high standard that it becomes very difficult to evaluate their intra-quality differences. Product users' judge manufactured goods not on a good-bad criterion, but on like- 
dislike preferences. For example Akita (1991), suggests that beauty and user-friendliness is more important than the sense of high technology within high-tech products, such as cameras, personal computers, and audio-visual equipment etc. Indeed Sipek (1993), goes as far to state that product designers have forgotten that their designed artifacts are made for people to use.

Potential users range widely, from the very young to the very old, men to women, healthy people to hospital patients, amateurs to professionals and so on. Therefore equipment should be designed to be adaptable, or in some cases specific to different peoples needs, in the most satisfying and efficient way for their personal use. This poses a new set of challenges for the designer in that a design proposal has to be evaluated at the concept stage of the design process, prior to detailed design, when s/he does not have a physical artifact, and no definite knowledge of how the market will respond to it, but simply a representation of it, for example; a design drawing or a 3D prototype model.

This challenge presents a new requirement to the work of design in that there is a need to create a methodology to evaluate designs more accurately and earlier in the design process (conceptual stage) that ideally has some universal characteristics. It is unlikely that there is a first law of design analogous to the first law of thermodynamics, but nonetheless there is a need for a procedure with a quantifiable result to guide the designer towards his goal of satisfying the needs, wants or desires of the user.

The paper presents a review of current progress in a research project, which is based on the work of Alexander (1964), in particular, who sought to introduce a generic methodology which could satisfy the needs of designers to describe and evaluate their designs. These basic ideas have been developed into a comprehensive methodology which has been instantiated into a framework for a Computer Aided Design Evaluation Tool (CADET), described further in Rodgers et al. (1993) and Rodgers et al. (1994).

Many authors including Ulrich and Seering (1988), Miles and Moore (1989), and Hollins and Pugh (1990) highlight the neglect of research activity into the early stages of product design and manufacture, for example the concept design evaluation stage. They suggest that this may be because concept design evaluation is generally subjective in nature, relying heavily on the knowledge, intuition and experience of designers and engineers and therefore does not readily lend itself to formal expression.

Cross (1994), suggests that although there may be many different models of the design process, they all have one thing in common - the need to improve on traditional methods of working in design. There are several reasons for this interest in developing new design methodologies, strategies and procedures, including for example: 
1. The fact that design problems designers have to solve nowadays has become extremely complex, for example industrial and plant machinery. Demands concerning materials and manufacturing processes' information, for example, is now so vast that it is well beyond the grasp of the individual designer to keep up to date.

2. Costs and investments involved in design projects are now so great, for example in setting-up of plant and machinery, purchase of raw materials, etc. that there are now greater pressures on the designer or design team to get it right first time before the project goes into production. Table 1 illustrates the costs involved throughout the various stages of the design of a new product.

Table 1: Costs involved in New Product Design (Hollins and Pugh, 1990).

$\begin{array}{ll}\text { Market research } & 6.9 \% \\ \text { Product design specification } & 5.5 \% \\ \text { Concept design } & 12 \% \\ \text { Detail design } & 17.5 \% \\ \text { Manufacturing } & 45.7 \% \\ \text { Selling } & 12.5 \%\end{array}$

3. The fact that the needs of end-users are perceived as having far more relevance nowadays, subsequently adding to the demands placed on designers (Heskett, 1992).

\section{Alexander's Design Model}

The presented method of product performance assessment is based on a formal model of the design process developed from that of Alexander (1964). Alexander developed his methodology in an attempt to help designers solve increasingly complex problems. Alexander highlighted the fact that the information required to solve even the simplest design problem is well beyond the limits of the individual designer. In an attempt to rectify this shortcoming designers tend more and more nowadays to rely on their intuition, personal experiences, gut-feeling and limited knowledge when making decisions throughout the various stages of product design. For example choosing materials, deciding product finishes, manufacturing methods to be utilised and so on. Alexander's main argument is that by relying on judgment and intuition alone, what is at best vague, but more often wholly inadequate. He asserts that by adopting logical structures to the process of design, this will result in making clear or explicit what is exactly involved or required in the design process. 
Alexander's design model is based on reducing the gap that exists between the designer and the user. The core of Alexander's approach is a formal description and representation of the design problem. The work presented here acknowledges the critiques that have been made of Alexander's work, by, for example, Lawson (1990). Lawson suggests that Alexander's work leads to a "rather mechanistic view" of design problems and illustrates this by pointing out two notions that are now commonly rejected:

1. That there exists an exhaustive set of requirements which can be listed at the start of the design process.

2. The listed requirements are all of equal value.

The work presented here directly addresses the second criticism of Alexander's method, specifically his listing of requirements being of equal value. It is fairly obvious that certain requirements of products are more important than others. This work incorporates this view by weighting the requirements and combining them, in turn, to give an overall measure of the product's performance.

In particular, this work is only utilising Alexander's analysis of the process, it is not utilising his suggested method of solution. The design model reported here is also extended to deal explicitly with mass produced manufactured items rather than 'one off' constructed items of architecture.

The design model utilises operators and entities to describe what are believed to be the fundamental actions and their objects respectively. Although the associated diagrams give the appearance of a procedural or flowchart model, they are not. They are intended to illustrate the abstract functional relationships between the various operators ${ }^{1}$.

\subsection{UNSELFCONSCIOUS PROCESS}

The ultimate objective of design is form. Every design problem commences with an attempt to attain 'fitness' between the two entities of: (i) the form, and (ii) its context. The form is the solution to the design problem; the context determines the design problem. Usually, in product design the actual goal for the designer is not the form alone, but the ensemble comprising the form and its context. 'Good fit' is a required characteristic of the ensemble. For example a kettle (form) should be able to rest safely on a work-surface (context) within a kitchen (ensemble) (Alexander, 1964: 15).

Alexander (1964: 48) describes the unselfconscious process as a type of built-in fixity-types of myth, tradition and taboo which oppose strong modification. Creators of form will only introduce modification under

\footnotetext{
${ }^{1}$ An abstract function defines the relationship between two sets independent of the actual set membership (Blyth 1975).
} 
sound compulsion where there are strong and obvious errors ("misfits") within the existing forms which demand correction.

In the unselfconscious process the designer operates directly on an actual form within its actual context. Determining the fit of form in context is by direct observation of the ensemble and in the absence of fit the design process determines actions to eliminate the misfits. An example of the unselfconscious process is the bespoke tailor fitting a suit to a client. The designer (bespoke tailor) will observe misfits in the ensemble of form (suit) and context (client) and make changes such as letting in or taking out seams. Formally the process is illustrated in Figure 1.

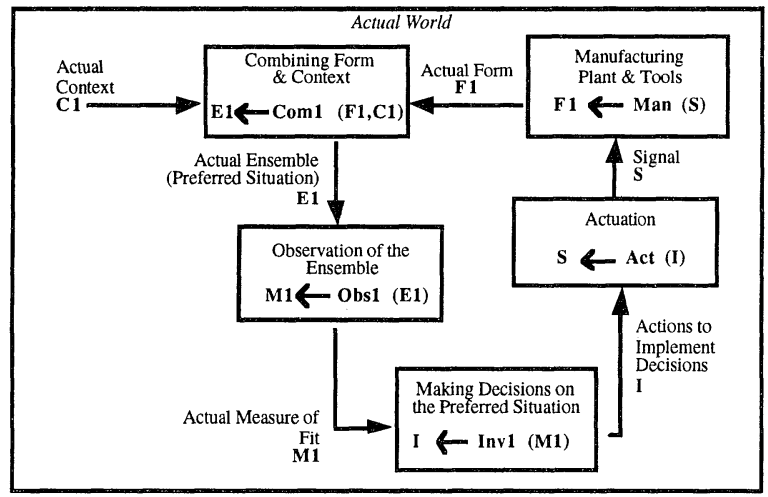

Figure 1. The unselfconscious process.

The nature of this abstract approach is that the work is not, at this stage, defining the content of entities or operators, but only the functional relationships between them. Figure 1 is explicitly not intended as a flowchart representation of the design process.

Actual Form F1 and actual context $\mathbf{C 1}$ are (real) entities combined by the operator Com 1 to create the ensemble entity E1. The designer observes the ensemble by operator Obs1 to determine the misfit entities M1. The designer then applies some cognitive process described by the operator Inv1 to determine the entity $\mathbf{I}$ of actions to be taken to eliminate the misfits. These actions are realised in the ensemble by actual tools or plant.

The actions are information in the memory of the designer which have to be converted into real physical events. This is done by a process of actuation described by the operator Act which converts information into power denoted by the entity $\mathbf{S}$. The entity $\mathbf{S}$ has both physical and informational significance in that it must be sufficient to cause an effect but controlled to produce the correct effect. For example the operator Act may describe an actuator such as a servo motor which (via other hardware) drives a machine tool from instructions $\mathbf{I}$ in the form of a part program. The output from the 
servo motor must supply sufficient power to cut material but with sufficient accuracy to cut it in the way required. The effect of $\mathbf{S}$ in producing a new form F1 depends on the actual machinery it connects to which is in turn described by the operator Man. These operators are intended to be complete and generic describing all processes required to generate the new form F1.

A more realistic model may be to describe Man as a differential operator, i.e. causing a change to an existing actual form rather than generating a completely new form. The given definition is used in the interests of simplicity later.

In the unselfconscious process actuation Act is predominately performed by the human mind in determining how tools should be operated or utilised. The unselfconscious designer need not be able to invent forms at all just respond to misfits (Alexander, 1964: 58). Most importantly the iterative modification of form to fit context occurs physically and is defined by the actual experience and satisfaction of the designer. Although an apparently obscure name the "Unselfconscious Process" is particularly apt. The designer is conscious of the form in context and responds directly to direct experience of the ensemble without consciously considering the change in form required to eliminate the misfit.

The observations made by the designer are determined by the physical configuration of the ensemble, which may be inorganic or organic, and the physical, social, cultural, and/or economic laws that apply to the configuration. Whilst the unselfconscious designer need have only intuitive knowledge of them, since he deals directly with the consequences, it will be seen later that they must be codified to produce rationalised predictions.

The unselfconscious approach is clearly unsuitable for industrially massproduced goods for the reasons cited by Jones (1980):

1. Specifying dimensions (form) in advance of manufacture makes it possible to split up the production work into separate pieces which can be made by different people. This is the 'division of labour' which is both the strength and the weakness of industrial society.

2. Initially this advantage of defining before making made possible the planning of things that were too big for a single craftsman to make on his own, for example, large ships and buildings. Only when critical dimensions have been fixed in advance can the works of many craftsmen be made to fit together.

3. The division of labour made possible by scale drawings can be used not only to increase the size of products but also to increase their rate of production. A product which a single craftsmen would take several days to make is split up into smaller standardised components that can be made simultaneously in hours or minutes by repetitive hand labour or by machine. 


\subsection{THE SELFCONSCIOUS PROCESS}

The method of form creation in the selfconscious process is very different from that in the unselfconscious process. Modifications are no longer made upon observation of error or misfit. They are, however, only made after a process of recognition and description by the specialist involved (Alexander, 1964: 55).

In the selfconscious process (Figure 2) the designer has become removed and is remote from the user of the product and the final physical product itself. Instead of being able to directly observe the ensemble, the designer investigates, explores and researches the actual context $\mathbf{C 1}$ and constructs a mental picture of it C2. This process is described by the operator Exp.

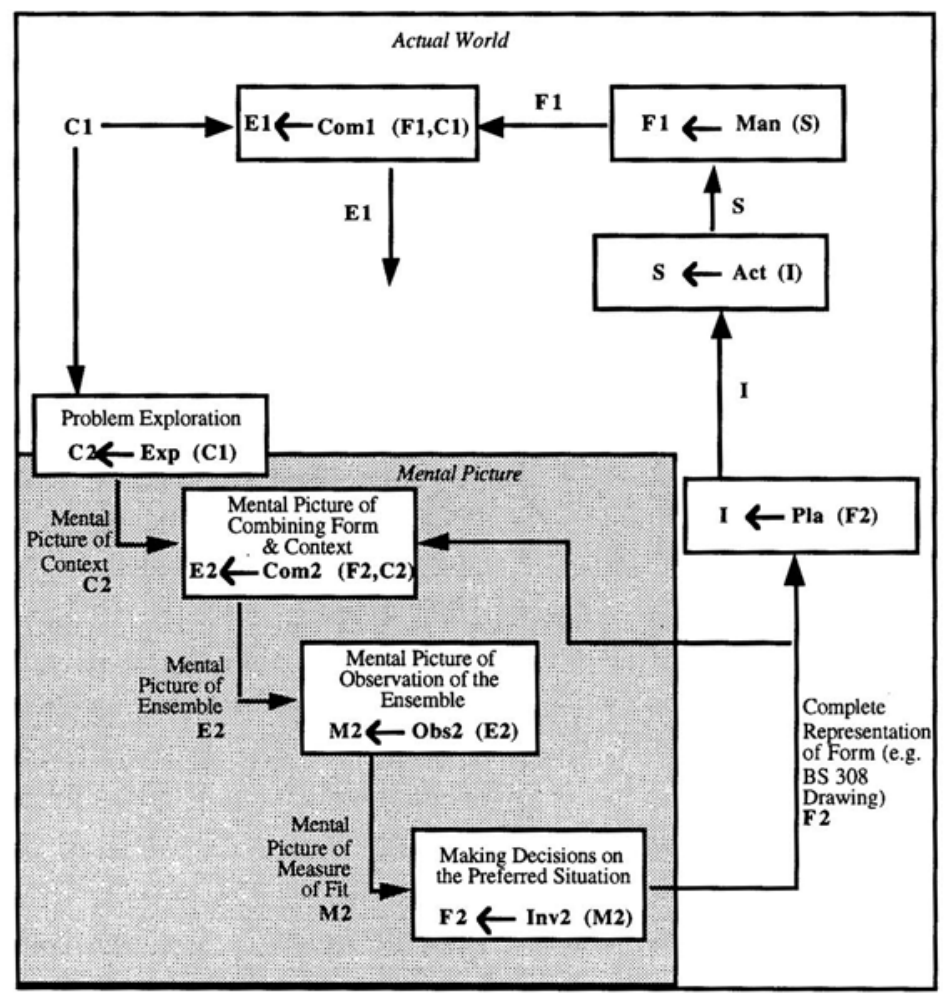

Figure 2. The selfconscious process.

Instead of working on the actual form the designer must work with a description or representation of it, F2, and through the operator Com 2 combine the form and context to make a mental picture of the ensemble E2. From this mental picture of the ensemble the designer attempts to estimate potential misfits M2. This process is described by the operator Obs2. In the 
same way as in the unselfconscious process the designer makes intuitive judgments, but this time on modifications to the form rather than actual actions to be taken. This process is described by the operator Inv2.

The process of inventing a form and physically realising it are separate. As Dormer (1993) indicates designers do not manufacture things. They think, they analyse, they may model or draw, and they specify. The most important distinction between the unselfconscious designer and the selfconscious designer is that the latter must define in detail complete and unambiguous descriptions of the shape, size, materials, and material finishes of the form prior to manufacture. Although designers may take manufacturing considerations into account there are further operations of manufacturing planning Pla, which determines the instructions I that will physically realise the form represented by $\mathbf{F 2}$, for example a BS 308 drawing (Parker, 1984).

The process of determining fit is a mental simulation of the observations the unselfconscious designer uses to determine his actions. Whilst the unselfconscious designer is dealing with reality the selfconscious designer is attempting to mentally predict a future reality.

\section{Representation of Form}

In the unselfconscious process the designer works directly with the physical form. In the selfconscious process he works on some representation of it. The representation is a complete definition of the shape, material and finish that the form will consist of ${ }^{2}$. When the actual form is realised measurements taken from it should correspond exactly to the measurements in the representation.

The representation of the form does not define a unique physical form. Because of inevitable tolerancing and measurement errors it defines a class of admissible actual physical forms and the actual form realised from it must be within that class.

\section{Misfits within the Mental Picture}

In the selfconscious process misfits are determined in part intuitively or by intuitively designed tests. In practice the selfconscious designer may well go through a process similar to that of the unselfconscious designer. In the example of the "off-the-peg" suit, the designer may well go through the same process of a series of successive changes or "fittings" with respect to

\footnotetext{
2 The representation may contain symbolic descriptions of standard components, e.g. (electrical or electronic) but these will always be supported by shape and material representations elsewhere.
} 
the standard mannequin as the bespoke tailor does with a client. However, unlike the bespoke tailor the result of identifying and eliminating misfits is not within the actual suit, but it is the cutting patterns representing the typical form of the suit. Although apparently identical the "off-the-peg" designer is undergoing a process of testing whilst the bespoke tailor is going through a process of production.

\section{Difficulties with the Selfconscious Process}

The operations defined within the selfconscious process are still predominately intuitive and imaginative. The designer uses drawings and diagrams to support the mental picture in his mind, however in that picture the decisions of fit of the proposed form within it are not clear. As stated initially, although the invention of form may well be intuitive, imaginative and not completely understood there is no reason why the fit of form with context should not be rationalised in an attempt to maintain the designer's intent. Alexander (1964: 77) addresses this problem by creating a formal picture of the mental picture by abstracting and defining its necessary features in formal terms. The fit of form with context can be formally defined in terms of the formal picture.

\subsection{FORMAL PROCESS}

Alexander asserts that within the selfconscious process the designer works entirely from the mental picture in his mind, and this picture is almost always wrong. He suggests eradicating this problem by constructing a formal picture of the design problem. This formal picture can then be scrutinised in a way not subject to the bias of language and experience (Alexander, 1964: 78). The formal picture is not intended to eliminate the intuitive and imaginative components of the design process, but to make it visible, discussable, open to criticism and make the designer accountable (Lawson, 1990).

Alexander defines the formal picture in terms of the observations of the form in context which could cause a misfit (Figure 3). The observations are called misfit variables which are either true or false. Alexander requires the selfconscious designer to state the criteria of the intuitive judgment of fit from the mental picture. Overall fit is the conjunction of the misfit variables. In the formal process the designer constructs a formal picture of the context $\mathbf{C 3}$ from the mental picture of the context $\mathbf{C 2}$. This process is described here by the operator For. The designer combines the formal picture of form $\mathbf{F 3}$ and context $\mathbf{C 3}$ to produce the formal picture of the ensemble E3. This process is described here by the operator Com3. The measure or quality of fit M3 is then determined through predicted observations of the ensemble E3. This process is described here by the operator Obs3. Synthesis of a new 
form F3 is created in response to the predicted measure of fit or misfit M3. This process is described here by the operator Inv3. The concept representation of the form F3 (e.g. drawing, annotated sketch, etc.) is then subject to a process of embodying the concept and adding greater detail which results in a complete and unambiguous description of the form $\mathbf{F} 2$ (e.g. BS 308 Drawings). The procedure from concept of form (F3) to detailed description of form (F2) generally follows along the following lines, (French, 1985):

1. Conceptual design

2. Embodiment design

3. Detail design

This process is described here by the operator Emb.

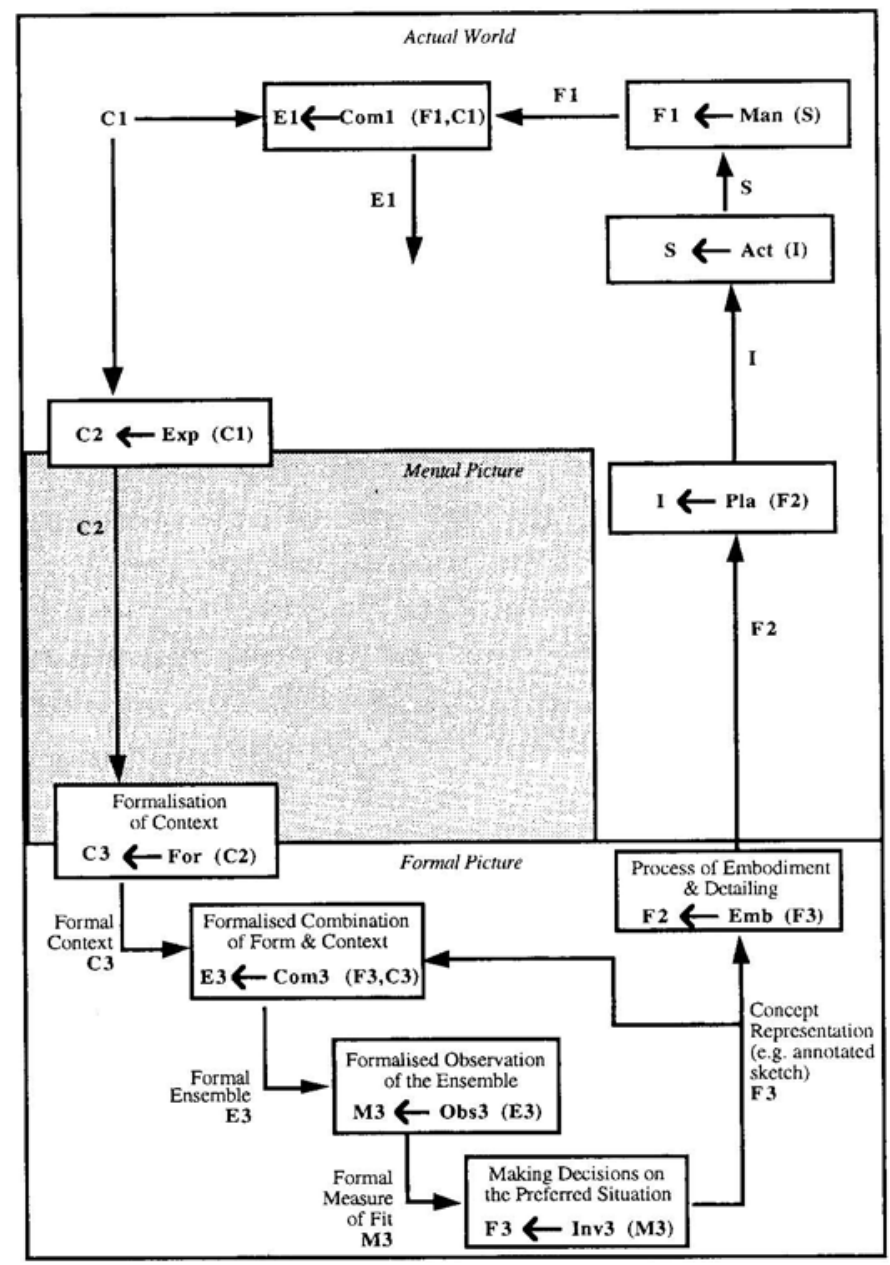

Figure 3. Formal picture of the mental picture. 
Determining the formal measure of fit M3 within the formal picture (Figure

3 ) is the process of product performance assessment at the conceptual stage of the design process.

\subsection{ATTRIBUTES AND ENSEMBLE}

\section{Misfit Variable}

Alexander's formalisation of context is based on a set of Boolean variables. However this implies that each requirement is of equal importance, a notion now widely rejected by many authors including Lawson (1990).

It is important to note that a misfit variable is defined as an observation that could be made of an actual ensemble. The paper will demonstrate later that the designer's problem at the conceptual stage of the design process is to predict this observation, since the actual ensemble does not exist.

To enable requirements of differing importance to be defined the misfit variable is extended to an attribute variable which can take on a wider range of values appropriate to the observation.

\section{Attributes}

An attribute a is defined by the set of values it may take which is defined as its type A.

$$
A=\{a: S(a)\}
$$

where $\mathrm{S}$ is an open sentence defining inclusion within A (Blyth, 1975).

$$
a \in A \text {, }
$$

with the constraint that A must be scalar and totally ordered.

Misfit variables (Alexander, 1964), are special cases of attribute variables equivalent to an enumerated type: \{false, true $\}$

The meaning or semantic of an attribute, for example 'consumes_fuel_ efficiently', is its method of observation from an actual ensemble. An attribute observation $\mathrm{Oba}$ is a function from an actual ensemble E1 to an attribute value $\mathrm{A}$.

$$
\text { Oba } \mid \mathbf{E 1} \rightarrow \mathrm{A}
$$

An attribute is the observation of an element of performance of an actual form F1 within an actual ensemble E1, i.e. the product in use. For example, a performance element of a car could be that it should consume fuel efficiently. The attribute 'consumes_fuel_efficiently', for example miles per gallon, could be directly measured under stated conditions. Another attribute such as 'looks_fast' would have to take values from an enumerated set such as $\{$ slow, average, good, quick, fast $\}$ but ultimately could only be measured from the stated responses of individuals. 
It is important that attributes are based on direct observation and do not implicitly contain theories about their causes. For example a suitcase may have an attribute 'comfortable_to_carry' which could be reasonably objectively defined and evaluated in terms of muscular discomfort. It should not however be defined in terms such as weight which implicitly reflect ergonomic theories of human capacity. Such considerations are clearly essential to the assessment but are not contained within definitions of attributes.

In general attributes range from the objective to the subjective (Figure 4).

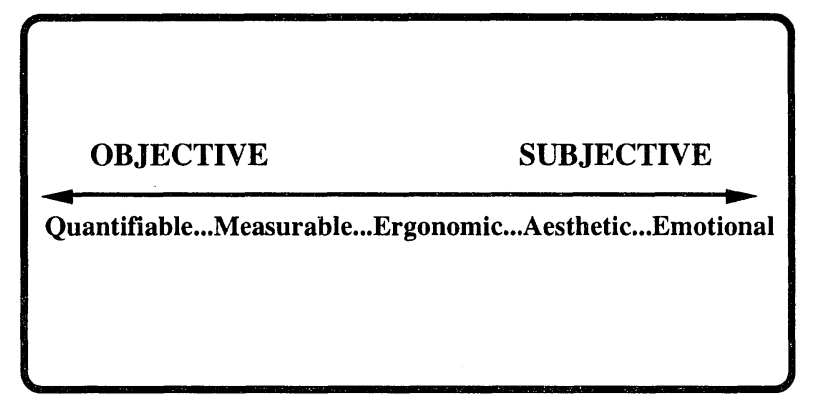

Figure 4. Attribute spectrum.

Definition of the attributes formalises the designer's view of the significant requirements of the form in use. All the attributes $\mathbf{A}$ for a form is the product set of the $\mathrm{n}$ individual attribute types defined,

$$
\mathbf{A}=\mathrm{A}_{1} \mathrm{X} \mathrm{A}_{2} \mathrm{X} \ldots . \mathrm{A}_{\mathrm{n}} \text {. }
$$

The elements of $\mathbf{A}$ are the $n$-tuples reflecting the evaluations of each attribute,

$$
\mathbf{a} \in \mathbf{A}, \quad \mathbf{a}=<\mathrm{a}_{1}, \mathrm{a}_{2}, \ldots . \mathrm{a}_{\mathrm{n}}>.
$$

The corresponding $\mathrm{n}$ attribute observation functions are,

$$
\mathrm{Oba}_{1}, \mathrm{Oba}_{2}, \ldots . \mathrm{Oba}_{\mathrm{n}} \text {, }
$$

and the single function which produces the attribute n-tuple is,

$$
\begin{aligned}
& \text { Oba } \mid \mathbf{E 1} \rightarrow \mathbf{A} \\
& \mathbf{a}=\left\langle\mathrm{Oba}_{1}(\mathbf{E} 1), \mathrm{Oba}_{2}(\mathbf{E} 1), \ldots . \mathrm{Oba}_{n}(\mathbf{E} 1)\right\rangle
\end{aligned}
$$

The attribute variables define the relevant aspects of the product in use.

\subsection{FORM}

The detailed representation of form $\mathbf{F} 2$ should be a complete and unambiguous representation of an actual form F1. However due to the inevitable measurement and manufacturing errors, F2 in practice defines a 
class of actual forms. F2 is in fact an inclusion condition for a class of admissible actual forms $\mathbf{F}$. The usual practice is to treat measurement as perfect and incorporate measurement error within the range of permissible actual forms. For example a component is measured and that measurement compared to a toleranced drawing. If the measurement is within tolerance then the component is, in that respect, within the class of admissible forms, otherwise it is not. It is not generally assumed that if a component is out of tolerance that it may still be within the class of admissible forms due to the measurement error.

This leads to algebraic complications since the manufacturing operator Man would have to be defined as producing classes of admissible actual forms rather than a single actual form. Consequently tolerancing and measurement error will be ignored and it will be assumed that there is a unique actual form $\mathbf{F 1}$ associated with its representation F2. The relationship between $\mathbf{F 1}$ and $\mathbf{F} 2$ is defined by a measurement operator Mes,

\section{Mes. I F1 $\rightarrow$ F2.}

Similarly it will be assumed that a concept representation $\mathbf{F 3}$ is associated with a unique actual form $\mathbf{F 1}$ also related by the operator Mes,

\section{Mes I F1 $\rightarrow$ F3.}

This of course implies that the process of embodiment, detailing Emb, and manufacture Man are completely deterministic for a given concept. This is clearly not the case in practice but makes little difference to the problem of product performance assessment at the concept stage of the design process.

\subsection{PERFORMANCE}

To get a single performance evaluation of the form in context the individual attributes must be combined in a way that reflects their relative importance (Lera, 1981). The final measure of performance $\mathbf{M 3}$ is the formal picture of the actual performance M1. The combination of attributes to find M3 is described by a combination function $\mathbf{C o b}$ which defines their relative importance.

$$
\text { Cob I A } \rightarrow \text { M3. }
$$

This addresses Lawson's (1990) major criticism of Alexander (1964) of not accounting for the relative importance of the attributes.

\subsection{CONTEXT}

The formalisation of context is the combination function Cob. Notice that defining the combination function implicitly defines the attributes as well as 
explicitly defining their relative importance. This definition is equivalent to Alexander's formalisation of context when all attributes are Boolean and their combination is conjunction. Notice again that the formalisation of context is defined in terms of observations that could be made of an actual ensemble.

\subsection{EVALUATION}

The definition of product performance is illustrated below (Figure 5).

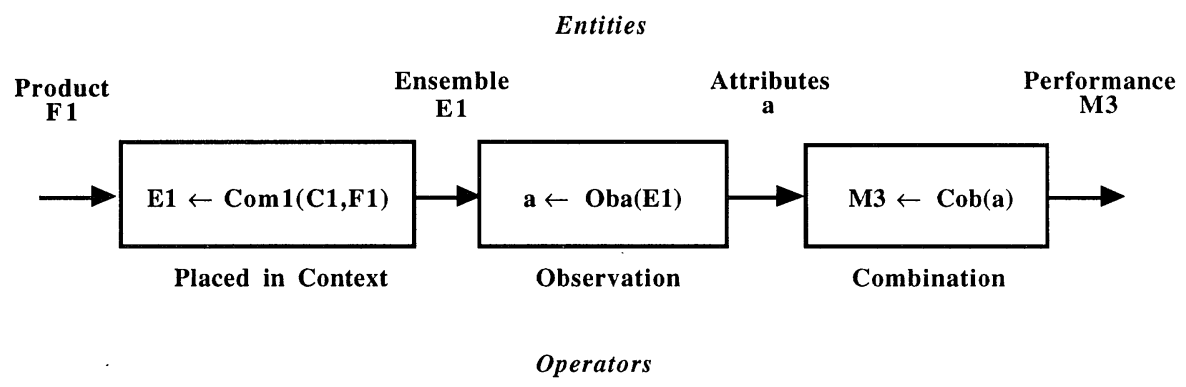

Figure 5. Performance assessment of an actual product.

The assessment of an actual product in context is by implementation of the operators Oba, which evaluates the attributes and $\mathbf{C o b}$, which combines them into a single performance assessment. However, at the conceptual stage of the design process there is no actual product. From Figure 3, M3 is predicted by the operator $\operatorname{Com} 3(\mathbf{C 3},) \cdot \mathbf{O b s} 3$. Consequently this operator is required to simulate $\mathbf{O b a} \cdot \mathbf{C o b}$.

\section{Product Performance Assessment}

\section{Definition of Assessment}

Product performance assessment at the conceptual stage was described as implementation of operators,

\section{Com3(C3,)・Obs3,}

on the concept representation of form $\mathbf{F 3}$ (see Figure 3).

The product performance assessment method has two elements of:

1. Problem Definition-definition of entity $\mathbf{C 3}$ by implementation of operator Exp•For

2. Evaluation -implementation of operators $\operatorname{Com} 3$ and $\mathbf{O b s} 3$ on $\mathbf{F} 3$ to produce the measure of performance M3. 
This work will start with the evaluation problem since the requirements of evaluation influence problem definition.

At the concept stage, where there is no actual product, the operator Oba must be simulated to predict the performance M3. This requires prediction of each individual attribute to be combined by the operator Cob. The operator Oba is simulated using two further operators Ext and Mod to be described later.

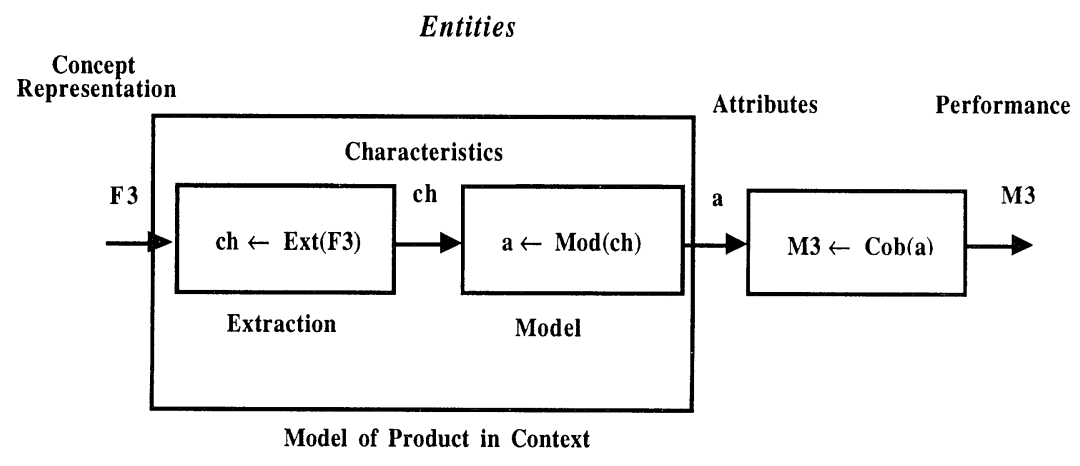

Operators

Figure 6. Performance assessment at concept representation stage.

\subsection{ATTRIBUTE PREDICTION}

The prediction of objective attributes such as: 'consumes_fuel_efficiently' is well defined, although in many cases may be mathematically difficult. The engineering sciences, for example thermodynamics, are predominately concerned with making these types of predictions, the results of which are embodied in available computer software, e.g. RASNA MECHANICA®.

Theoretical models for predicting behaviour are based not of the complete form itself but on specific properties extracted from it. In many cases the same property will appear in more than one attribute. It is therefore convenient and expedient to decompose the operator Obs3 into a composition of a further operator of extraction Ext, a model Mod, and a new entity reflecting the extracted properties called characteristics $\mathbf{C h}$.

Characteristics are inherent properties of any product, independent of the product's use, and can be determined purely from the representation. Product characteristic examples include mass, colour, material specifications, dimensional information (length, width, height, etc.).

Characteristics have the same mathematical structure as attributes. A characteristic ch is defined by the set of values it may take which is defined 
as its type $\mathrm{Ch}$.

$$
\mathrm{Ch}=\{\mathrm{ch}: \mathrm{S}(\mathrm{ch})\}
$$

where $S$ is an open sentence defining inclusion within Ch (Blyth, 1975).

$$
\mathrm{ch} \in \mathrm{Ch} \text {, }
$$

The characteristics of a form $\mathbf{C h}$ are the product set of the $\mathrm{n}$ individual characteristic types defined for the form,

$$
\mathbf{C h}=\mathrm{Ch}_{1} \mathrm{X} \mathrm{Ch} 2 \mathrm{X} \ldots \mathrm{Ch}_{\mathrm{n}} .
$$

Consequently the elements of $\mathbf{C h}$ are the n-tuples reflecting the determination of each characteristic,

$$
\text { ch } \in \mathbf{C h}, \mathbf{C h}=<\mathrm{ch}_{1}, \mathrm{ch}_{2}, \ldots . . \mathrm{ch}_{\mathrm{n}}>.
$$

The operator Ext is a function from form $\mathbf{F 3}$ to characteristics $\mathbf{C h}$.

$$
\text { Ext } \mid \mathbf{F 3} \rightarrow \mathbf{C h}
$$

and the model Mod a function from characteristics $\mathbf{C h}$ to attributes $\mathbf{A}$.

$$
\operatorname{Mod} \mid \mathbf{C h} \rightarrow \mathbf{A} \text {. }
$$

\subsection{ATTRIBUTE EVALUATION EXAMPLE}

The assessment method, developed within this work, is illustrated by the following example. The example will outline the assessment of a single attribute.

\subsection{TOOTHBRUSH}

In this example the concept representation $\mathbf{F 3}$ is an annotated sketch (Figure 7). In this case the attribute is objective but less easy to define since one has had to use natural language to describe the observation rather than mathematical language representing specific observations.

Attribute

reaches_all_teeth $\in\{$ True, False $\}$

Method of Observation

reaches_all_teeth $=$ filament ends contact with every tooth surface in the mouth".

In this example natural language formulation has been used and is denoted within double quotation marks. 


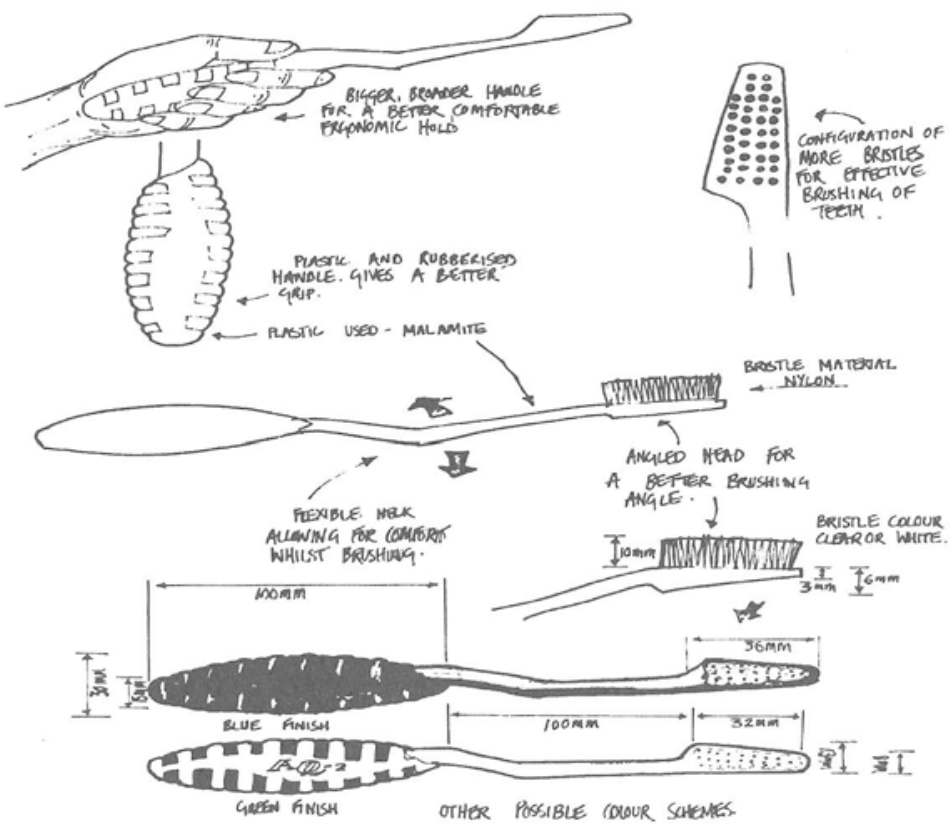

Figure 7. Design sketch of toothbrush.

\section{Model}

The model is constructed from considerations of spatial occupancy of the toothbrush drawn from computer-generated three-dimensional models representing the access region in the mouth for a given brushing action drawn from expert knowledge (Walsh and Lamb, 1992/93).

In this case the model was constructed empirically by using a plaster cast of teeth and estimates of cheek flexibility to determine the region Access (Figure 8 ). The sweep trajectory $\mathbf{M}$ is a conservative approximation to ideal brushing action determined from interviews from dentists (Walsh and Lamb, 1993). The model is expressed as :

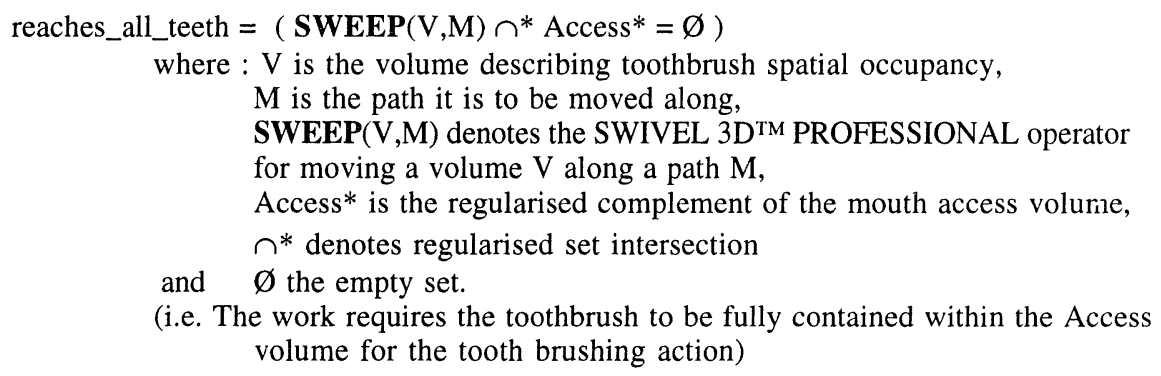

(i.e. The work requires the toothbrush to be fully contained within the Access volume for the tooth brushing action)

\section{Characteristics}

$\mathbf{V}$ is the volume representing the spatial occupancy of the toothbrush. 


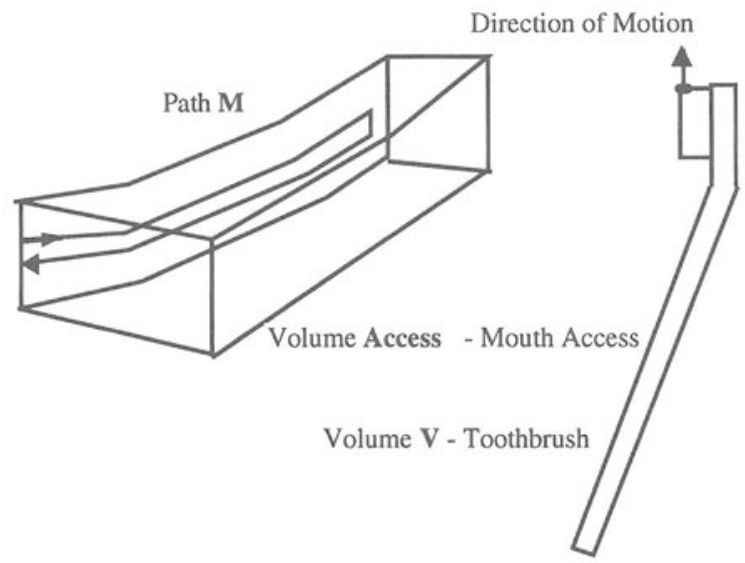

Figure 8. Diagram of access volume within mouth (empirically based model).

\section{Attribute Evaluation}

A $=\{<$ reaches_all_teeth $>$ reaches_all_teeth $\in\{$ true,false $\}\}$,

Oba = "filament ends contact with every tooth surface in the mouth".

$\mathbf{C h}=\{\langle\mathrm{V}\rangle \mid \mathrm{V} \in$ bounded regular subsets of $\mathbf{E 3}\}$, $\mathbf{c h}=\mathbf{E x t}(\mathbf{F} \mathbf{3})=$ "Construction of $\mathrm{V}$ in SWIVEL 3D ${ }^{\mathrm{TM}}$ PROFESSIONAL", $\operatorname{Mod}(\mathbf{c h})=$ "Definition of M and Access within SWIVEL 3D ${ }^{\mathrm{TM}}$ PROFESSIONAL", $\mathbf{a}=$ "Execution of the Sweep operator in SWIVEL 3D"T PROFESSIONAL and visual $\mathbf{a}=$ True inspection of the generated image (Figure 9)"

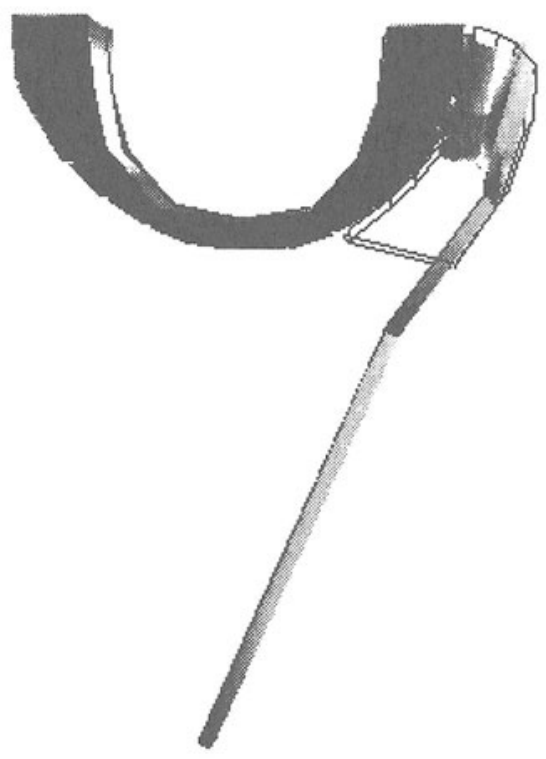

Figure 9. SWIVEL 3D ${ }^{\mathrm{TM}}$ PROFESSIONAL generated model of toothbrush in mouth. 


\subsection{CRITIQUE OF WORKED EXAMPLE}

The example illustrates the method of assessment. The example shows the organisation of information embedded within the assessment system. The system contains the following elements:

\section{Association between User and Designer Views}

An attribute is a formalisation of the intuitive judgment that would be made by the designer, or in other words the designer's "feel" for the problem, based on the requirements of the user. However, disagreement and misunderstanding can, and does, arise between the designer and the user (of the product, system, etc.). Cross (1994), states that this is because the designer and the user focus on different aspects of the product's requirements. The user generally focuses attention on the attributes of the product and states his or her requirements in natural terms, for example 'easy_to_clean'. The designer, however, concentrates more on the product's characteristics, which seek to establish the product attributes, which in turn attempt to satisfy the users' requirements. This approach addresses the problem by formally linking the physical characteristics of the product to a clear statement of the user requirements. For example, in the case of the toothbrush, the attribute and its observation,

\section{reaches_all_teeth $=$ "filament ends contact with every tooth surface in the mouth".}

clearly reflects the user requirement whilst the model links the relevant characteristics of spatial occupancy, which are under the control of the designer, to it.

\section{Attribute Selection}

It is questionable whether a complete list of requirements (attributes) can be defined for a product at the start of the design process. Many requirements of products become apparent only through the actual process of assessing design proposals.

\section{Model}

Whilst the problem of attribute selection is in determining an adequate, even if incomplete, set of attributes, the potential difficulty in the model is in accurately simulating those attributes that are defined. Moreover, the model itself, (i.e. the equation) says little to the designer about the rationale for its construction, for instance: the toothbrush example is based on ergonomics and human factors theory. If such a system is to be of use to the designer then the rationale must also be available in an explanation facility. 


\section{Attribute Type}

The assessment of the attribute in the example was to predict whether the form proposed would be suitable, for example would the toothbrush 'reach_all_teeth'. The assessment of this example was either true or false, in other words Boolean. However, it would be more useful to facilitate the designer with a numerical result that rates or scores the attributes. This would then assist the designer to address the specific characteristics that failed.

\section{FLEX Implementation of the Evaluation Methodology}

A computer implementation of the assessment method has been written in FLEX - an expert system toolkit that offers frame based, data driven and rule based functionality fully integrated into a PROLOG environment.

The computerisation of the assessment methodology (Figure 10) requires implementation of exactly the same operators used in the toothbrush example previously.

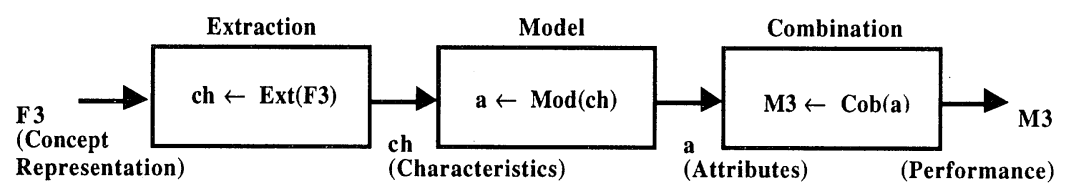

Figure 10. CADET tool implementation requirements of Obs3.

FLEX implementation of the previous defined operators, Ext, Mod, and Cob is required. The implementations are illustrated using the performance of an actual product, i.e. a toothbrush, as an example. In this example the attributes of the toothbrush and their methods of observation are:

A1: long_lasting $\in\{0 . .100\}$

Oba1: long_lasting $=$ "lasts a minimum of three months without wearing out"

A2: comfortable_to_hold $\in\{0 . .100\}$

Oba2: comfortable_to_hold = "comfortable to hold whilst brushing teeth"

A3: removes_plaque_efficiently $\in\{0 . .100\}$

Oba3: removes_plaque_efficiently $=$ "removes enough plaque each time to prevent a significant amount of tooth decay"

A4: does_not_irritate_gums $\in\{0 . .100\}$

Oba4: does_not_irritate_gums = "doesn't make gums bleed or cause sore gums" 
A5: reaches_all_teeth $\in\{0 . .100\}$

Oba5: reaches_all_teeth $=$ "filament ends contact with every tooth surface in the mouth"

A6: looks_attractive $\in\{0 . .100\}$

Oba6 : looks_attractive $=$ "looks attractive within a bathroom environment"

The attribute set for this problem is:

$$
\begin{aligned}
& \mathbf{A}=\mathrm{A} 1 \mathrm{X} \text { A2 X A3 X A4 X A5 X A6, } \\
& \mathbf{a} \in \mathbf{A}=<\text { long_lasting,comfortable_to_hold,removes_plaque _ }
\end{aligned}
$$

and the corresponding observation set,

\{ "lasts a minimum of three months without wearing out", "comfortable to hold whilst brushing teeth", "removes enough plaque each time to prevent a significant amount of tooth decay", "doesn't make gums bleed or cause sore gums", "filament ends contact with every tooth surface in the mouth", "looks attractive within a bathroom environment"\}.

The combination function in this case is a linear weighting of the attributes,

$\operatorname{Cob}(\mathrm{a})=\Sigma_{1 \leq \mathrm{i} \leq 6} \mathrm{w}_{\mathrm{i}} \mathrm{a}_{\mathrm{i}}$.

\subsection{FLEX IMPLEMENTATION OF OPERATOR COB}

Operator Cob is implemented by the FLEX structure action. Within this structure each attribute is identified by a FLEX relation identifier of the same name (Figure 11).

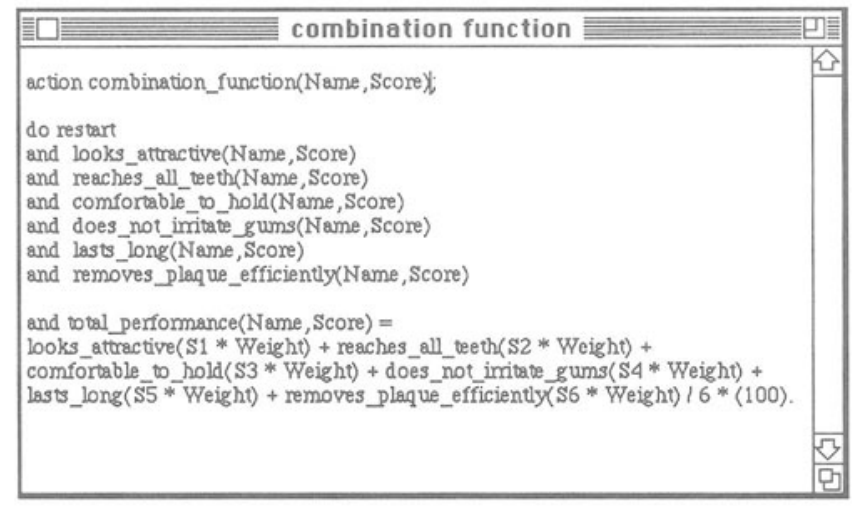

Figure 11. Combination function. 


\subsection{FLEX IMPLEMENTATION OF OPERATOR MOD}

The model for each of the attributes in the combination function is coded as a relation in FLEX based on knowledge extracted from experts, for example Walsh and Lamb (1992/93), and Delaunay (1982).

For example the model for the attribute 'does_not_irritate_gums' is a model of the observation "doesn't make gums bleed or cause sore gums" and is shown below (Figure 12).

i* DOES NOT IRRITATE GUMS CODE *I
relation does_not_initate_gums(Name,Score)
if Name is an instance of toothbrush
if the head_length of Name is greater than 18 and the
head_length of Name is less than 31
if the head_width of Name is greater than 6 and the
head_width of Name is less than 13
if the filament_diameter of Name is greater than 0.15 and the
filament_diameter of Name is less than 0.31
if the filament_material of Name is some instance of
non_imitate_oral_material
if the toothbrush_material of Name is some instance of
non_imitate_oral_material
if the head_shape of Name is some instance of
non_iritate_head_shape
if the head_cross_section of Name is some instance of
non_imitate_section

Figure 12. 'does_not_irritate_gums' model.

The model consists of a collection of clauses based on either the product characteristics or sub-relations. For example, the first clause, if Name is an instance of toothbrush', ties the attribute to the class of product defined as toothbrushes. The second clause, 'if the head_length of Name is greater than 18 and the head_length of Name is less than 31', is directly based on the product characteristic 'head_length' and reflects expert opinion (Chong and Beech, 1983). The fifth clause, 'if the filament_material of Name is some instance of \{non_irritate_oral_material\}', is based on the sub-relation ' $\{$ non_irritate_oral_ material\}'. This sub-relation is intended to be applicable to any item which is placed in the mouth and forms part of a library of similar sub-relations. This is implemented in FLEX by the following code shown below (Figure 13). 


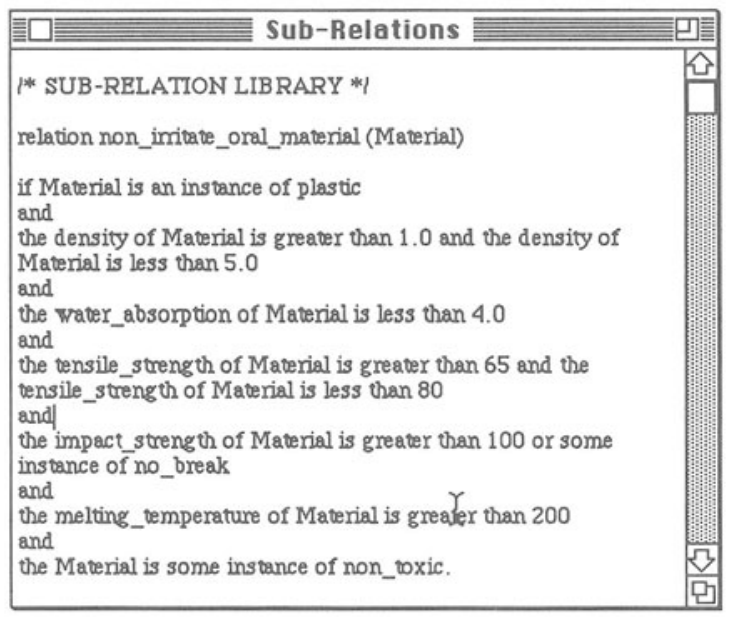

Figure 13. CADET tool library of sub-relations.

Each attribute has a similar model and their combination is the FLEX implementation of the operator Mod.

\subsection{FLEX IMPLEMENTATION OF OPERATOR EXT}

Each of the attribute models defines the characteristics necessary for its computation. The list of characteristics required are shown below (Figure 14). Notice that certain product characteristics such as 'filament_diameter', 'handle_cross_section' and 'head_shape' occur in more than one attribute model. The process of extraction is manual. The designer must inspect what ever concept representation $\mathrm{s} / \mathrm{he}$ is assessing in response to system prompts. In principle however if the system was linked to a CAD system then the type of feature extraction facilities available on some systems could be exploited either to automatically extract the characteristics or at least provide user friendly interactive methods, analogous to the systems described by Tovey (1994) and Buck (1992/93).

\section{Example of the CADET System in Use}

The CADET system may be used for either a total evaluation or for individual attribute evaluations. Each attribute can now be computed by selecting it from the pull down menu (Figure 15). The designer is requested to fill in the product characteristics describing his or her concept design proposal at the CADET system dialog box prompt (Figure 16), in this case actual characteristics of the toothbrush concept design proposed, e.g. 'toothbrush_length','handle_thickness', 'handle_cross_section', etc. 


\begin{tabular}{|c|c|c|c|c|c|c|}
\hline $\begin{array}{c}\text { Product } \\
\text { Characteristics }\end{array}$ & 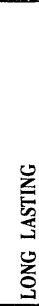 & 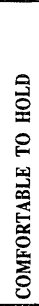 & 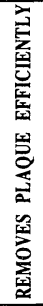 & 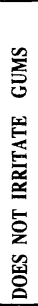 & 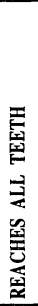 & 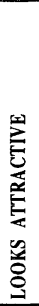 \\
\hline Handle Length & & $\mathbf{X}$ & & & & \\
\hline Toothbrush Length & & $\mathbf{X}$ & & & $\mathbf{X}$ & \\
\hline Head Length & & & & $\mathbf{X}$ & $\mathbf{X}$ & \\
\hline Filament Length & $\mathbf{x}$ & & $\mathbf{x}$ & & $\mathbf{X}$ & \\
\hline Filament Diameter & $\mathbf{x}$ & & $\overline{\mathbf{X}}$ & $\mathbf{x}$ & & \\
\hline Handle Width & & $\overline{\mathbf{X}}$ & & & & \\
\hline Head Width & & & & $\overline{\mathbf{X}}$ & & \\
\hline Handle Thickness & & $\mathbf{x}$ & & & & \\
\hline Head Thickness & & & & & & \\
\hline $\begin{array}{c}\text { Number of Filaments in One } \\
\text { Tuft (Packing Density) }\end{array}$ & $\mathbf{x}$ & & & & & \\
\hline \multicolumn{7}{|l|}{ Number of Tufts in Head } \\
\hline Filament Material & $\mathbf{x}$ & & $\mathbf{x}$ & $\mathbf{x}$ & & \\
\hline Toothbrush Material & $\overline{\mathbf{x}}$ & & & $\overline{\mathbf{X}}$ & & $\mathbf{X}$ \\
\hline Head Shape & & & & $\overline{\mathbf{X}}$ & $\overline{\mathbf{X}}$ & $\overline{\mathbf{X}}$ \\
\hline Handle Shape & & $\mathbf{X}$ & $\mathbf{X}$ & & & $\mathbf{X}$ \\
\hline Filament-End Shape & $\mathbf{x}$ & & & & & \\
\hline Handle Cross-Section & & $\mathbf{X}$ & & & & $\mathbf{x}$ \\
\hline Head Cross-Section & & & & $\mathbf{x}$ & & \\
\hline Tuft Arrangement & & & $\mathbf{x}$ & & & \\
\hline Toothbrush Colour(s) & & & & & & $\mathbf{x}$ \\
\hline Filament Colour(s) & & & & & & $\mathbf{x}$ \\
\hline Toothbrush Finish & & $\overline{\mathbf{X}}$ & & & & \\
\hline $\begin{array}{r}\text { Angle between Toothbrush } \\
\text { Head \& Handle }\end{array}$ & & & $\mathbf{x}$ & & $\mathbf{x}$ & \\
\hline
\end{tabular}

Figure 14. Selection of attributes with product characteristics required to construct each model.

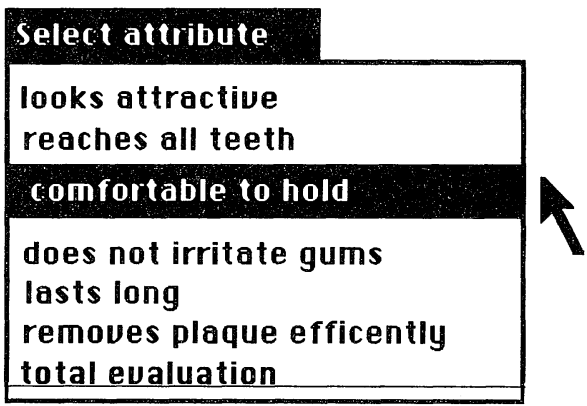

Figure 15. Attribute selection menu. 


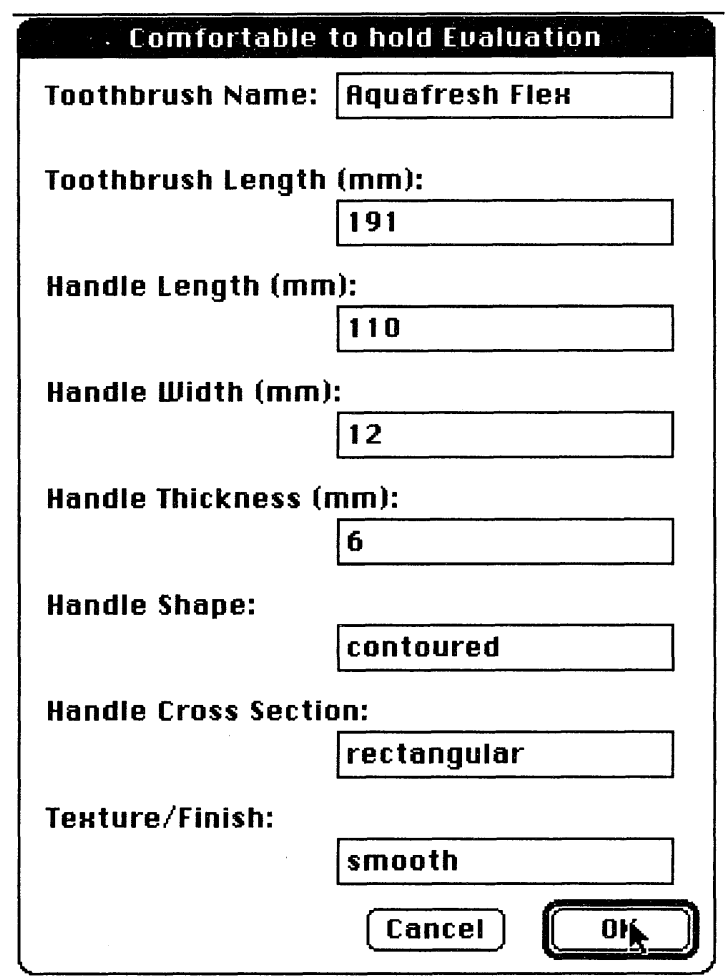

Figure 16. CADET system product characteristics extraction dialog.

Notice that the system obliges the designer to have defined sufficient detail for the concept to be evaluated. Having entered the product characteristic data into the system the designer can then quickly evaluate the potential for success of his or her concept design proposal.

The result is displayed which gives the designer a numerical indication of how well or how badly the concept proposed has done (Figure 17).

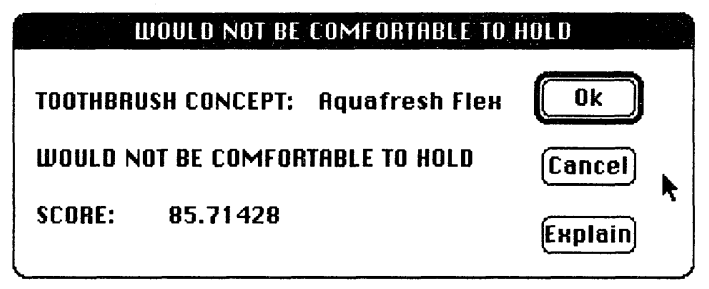

Figure 17. CADET system evaluation dialog.

The designer may investigate the reasons for the evaluation by referring back to the FLEX relations previously described. However as was found in 
the previous examples whilst the FLEX language makes the calculations clear the underlying rational is not apparent. To achieve this an explanation facility containing the expert knowledge used is available for each attribute (Figure 18).

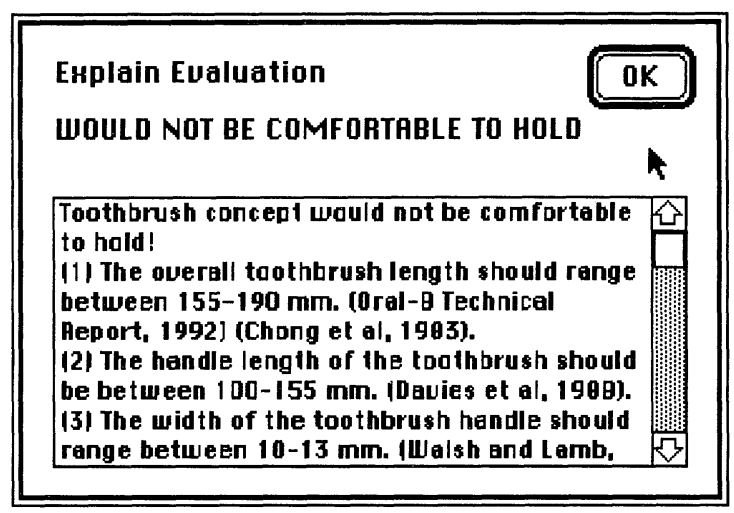

Figure 18. CADET system explanation facility.

\section{Conclusions}

The paper has presented a new methodology for assessing product performance in user rather than product terms. The methodology developed addresses the problem of predicting the measure of fit of product design proposals at the conceptual stage of the design process.

The method of assessment is made up of three major stages:

1. Defining the attributes, in users' terms.

2. Determining the model of each attribute.

3. Defining the combination function.

The intention for the future is to consolidate and further verify this work by introducing the following features:

\section{Verification of CADET System Testing}

The CADET System evaluation results will be tested against conclusions drawn from conducting appropriately controlled experiments on different groups of parties, for example product designers, manufacturers and users. These tests will seek to determine if:

(a) The evaluations are equivalent

(b) They are equivalent for the same reasons as those embodied within the system

or otherwise. 
Formally in terms of the system description the tests will seek to establish,

(a) If the combination function Cob contains sufficient and relevant attributes

(b) If the operator Mod is an accurate model of the observations it is intended to predict.

\section{Assessment of Other Products}

A great deal of design work in practice is concerned not with the creation of radical new design concepts but with the making of modifications to existing product designs. These modifications seek to improve a product-to improve its performance, to enhance its appearance, and so on.

The prototype CADET System is at present being tested against established product designs, particularly consumer products whose purpose and use is well defined, for example: telephones, electric kettles, electric hairdriers, system and disposable shavers. This type of product has evolved through several generations of products as end-user needs have developed.

\section{Design Model Development}

For the designer the CADET assessment of product performance is an optimisation criteria s/he is working towards, i.e. s/he is attempting to maximise the rated performance of the concept. The design model developed so far has deliberately not had a chronological element, it has only demonstrated the relationship between defined operations. It is intended to develop this model into a chronological model by treating it as an optimisation problem of performance. It is also anticipated that the design process will be described by some form of genetic algorithm within this.

\section{References}

Akita, M.: 1991, Design and ergonomics, Ergonomics, 34(6), 815-824

Alexander, C.: 1964, Notes on the Synthesis of Form, Harvard University Press, Cambridge, Massachusetts.

Blyth, T.S.: 1975, Set Theory and Abstract Algebra, Longman, London.

Buck, P.: 1992-93, Providing intelligent support for design, Manufacturing Intelligence, Winter, 10-13.

Chong, M. P. and Beech, D. R.: 1983, Characteristics of toothbrushes, Australian Dental Journal, 28(4), 202-211

Cross, N.: 1994, Engineering Design Methods: Strategies for Product Design (2nd edn), John Wiley, Chichester.

Delaunay, P.: 1982, Some current data involved in the choice of toothbrush, Actualites Odonto-Stomatologiques, 138, 249-258.

Dormer, P.: 1993, Design Since 1945, Thames and Hudson, London.

French, M. J.: 1985, Conceptual Design for Engineers (2nd edn), The Design Council, London.

Heskett, J.: 1992, Product integrity, Innovation, Spring, 17-19. 
Hollins, B. and Pugh, S.: 1990, Successful Product Design: What to do and when, Butterworth, London.

Japan Industrial Design Promotion Organization (ed.): 1990, Good Design Products 1990, JIDPO, Japan.

Jones, J. C.: 1980 edn, Design Methods: Seeds of human futures, John Wiley, Chichester.

Lawson, B.: 1990, How Designers Think-The Design Process Demystified (2nd edn), Butterworth Architecture, London.

Lera, S. G.: 1981, Architectural designers' values and the evaluation of their designs, Design Studies, 2(3), 131-137.

Miles, J. C. and Moore, C. J.: 1989, An expert system for the conceptual design of bridges, Proceedings of the Artificial Intelligence in Civil and Structural Engineering Conference, pp. 171-176.

Parker, M. (ed.): 1984, Manual of British Standards in Engineering Drawing and Design, British Standards Institution/Hutchinson, London.

Rodgers, P. A., Patterson, A. C. and Wilson, D. R.: 1993, A computer-aided evaluation system for assessing design concepts, Manufacturing Intelligence, 15, 15-17.

Rodgers, P. A., Patterson, A. C. and Wilson, D. R.: 1994, Evaluating the relationship between product and user, IEE Computing and Control Division Colloquium on Customer Driven Quality in Product Design, Digest No: 1994/086.

Sipek, B. (ed.): 1993, The International Design Yearbook, Laurence King, New York.

Tovey, M.: 1994, Form creation techniques for automotive CAD, Design Studies, 15(1), 85114.

Ulrich, K. and Seering, W.: 1988, Computation and conceptual design, Robotics and Computer-Integrated Manufacturing, 4(3/4), 309-315.

Walsh, T. F. and Lamb, D. J.: 1992/93, Update of oral hygiene aids: Toothbrushes, Dental Health, 31(6), 3-5.

Walsh, T. F. and Lamb, D. J.: 1993, Research visit to conduct knowledge engineering exercise with T. F. Walsh and D. J. Lamb, Department of Restorative Dentistry, School of Clinical Dentistry, Sheffield University Dental School, 19 March. 